\title{
Commentary: The straw that broke the camel's back
}

\author{
Riyad Karmy-Jones, MD, FACS, FRCSC
}

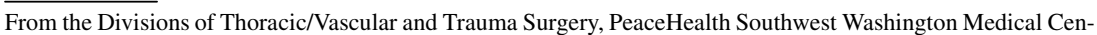
ter, Vancouver, Wash.

Disclosures: Author has nothing to disclose with regard to commercial support.

Received for publication July 15, 2019; accepted for publication July 17, 2019; available ahead of print July 25, 2019.

Address for reprints: Riyad Karmy-Jones, MD, FACS, FRCSC, Divisions of Thoracic/Vascular and Trauma Surgery, Peace Health Southwest Washington Medical Center, 505 NE 87th Ave, Building B, Suite 301, Vancouver, WA 98664 (E-mail: rkarmy-jones@ peachealth.org).

J Thorac Cardiovasc Surg 2020;160:654-5

$0022-5223 / \$ 36.00$

Copyright (c) 2019 by The American Association for Thoracic Surgery

https://doi.org/10.1016/j.jtcvs.2019.07.025

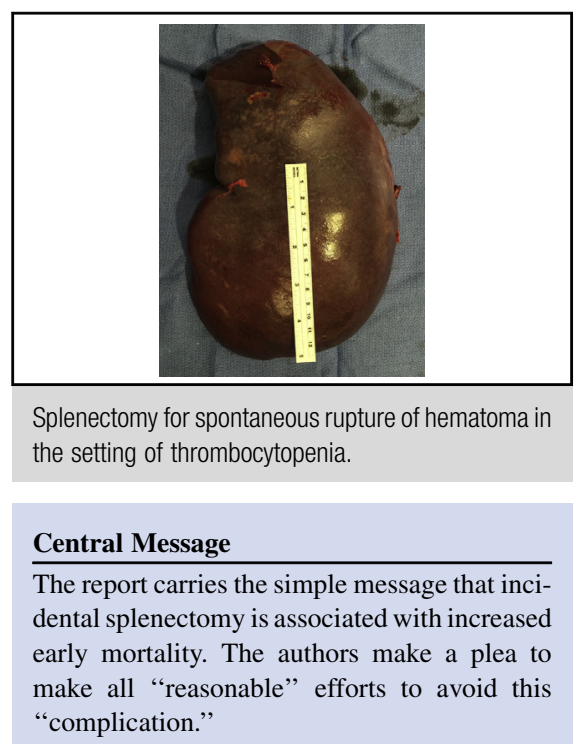

See Article page 641
According to Wikipedia, ${ }^{1}$ the idiom of the final straw refers to "the seemingly minor or routine action that causes an unpredictably large and sudden reaction, because of the cumulative effect of small actions." Each specialty has a classic complex operation that requires many things to go right, and certainly repair of thoracoabdominal aneurysms can be grouped with these. The report in this issue of the Journal by Chatterjee and colleagues ${ }^{2}$ carries the simple message that incidental splenectomy in this setting is associated with increased early mortality, to which they add a plea to make all "reasonable" efforts to avoid this "complication."

Splenectomy has been associated with a number of complications, including gastric distention, pancreatitis, left lower lobe collapse, and either direct or indirect immunosuppression, with increased risk of septic complications. Chatterjee and colleagues ${ }^{2}$ did note significant increases in the incidences of sepsis, respiratory failure, gastrointestinal complications, and need for reoperation for bleeding in the incidental splenectomy group. The differences were not so marked in the propensity-matched analysis, but Chatterjee and colleagues ${ }^{2}$ suggest that their numbers may not have been sufficiently powered.

Chatterjee and colleagues ${ }^{2}$ do not dwell in depth on specific techniques to avoid this, but it is telling that the incidence is increased in emergency, reoperative, and extent 2 surgery. The risk of splenic injury has been linked in other fields to all of these, including nephrectomy for locally advanced tumors.

Typical causes of splenic injury would be retractor injury or tearing of the capsule as a result of adhesions. In the illustration provided, it looks as if the spleen has been exposed outside the peritoneum, but in many cases the retroperitoneum can be reflected intact, which makes the diagnosis of a splenic injury more problematic. If a splenic injury is noted before heparinization or after aortic reconstruction, the question is how safe it to is "pack it off."

Chatterjee and colleagues ${ }^{2}$ stress prevention of injury to the spleen. This could include very careful mobilization and lysis of adhesions, as well as gentle retraction, perhaps with padded instruments. Typically, dense adhesions can form between the spleen and diaphragm, which might require taking down with coagulation or staple devices. The other adhesions may really be short, dense, tense natural ligaments to the stomach, kidney, or retroperitoneum, which should be managed in a similar fashion. Unfortunately, before systemic heparinization and cooling, even topical agents to control small injuries may not be sufficient.

After completion of aortic repair, the decision of whether to attempt splenic salvage is still a difficult one. Unlike the typical abdominal surgery, the spleen is not easily "packed off," and given the incision, it is not easy to consider a relaparotomy. In addition, some of the more effective "combat closure pads" require removal at a second operation, generally within 24 hours. A number of salvage techniques have been tried, including ligating the splenic artery, but each carries its own unique risk. Probably, in the setting of minor injury, the optimal approach would be to use topical, absorbable hemostatic agents or argon beam.

It is worthwhile to consider the technique of splenectomy. The spleen can be removed as close to the splenic mass as possible, lowering the risk of inadvertent pancreatic injury. The short gastric arteries should be ligated as far away from the gastric wall as possible. A simple technique 
is to use vascular staples. Inattention to detail can lead to gastric or pancreatic injury, as well as missing small collateral arteries.

Unfortunately, as the article of Chatterjee and colleagues $^{2}$ shows, even in the best hands a surgery of this complexity can be hostage to "the final straw."

\section{References}

1. Straw that broke the camel's back. Wikipedia. Available at: https:// en.wikipedia.org/wiki/Straw_that_broke_the_camel\%27s_back. Accessed August 19, 2019.

2. Chatterjee S, LeMaire SA, Green SY, Price MD, Hiruni S, Amarasekara HS, et al Is incidental splenectomy during thoracoabdominal aortic aneurysm repair associated with reduced survival? J Thorac Cardiovasc Surg. 2020;160:641-52.e2. 